\title{
DISKUSSION
}

DDS - Die Deutsche Schule

110. Jahrgang 2018, Heft 4, S. 369-382

https://doi.org/10.31244/dds.2018.04.07

(C) 2018 Waxmann

Martin Rothland

\section{Allgemeine Didaktik und empirische Unterrichts- forschung als Teilgebiete der Schulpädagogik}

\begin{abstract}
Zusammenfassung
Allgemeine Didaktik und empirische (quantitative) Unterrichtsforschung der Schulpädagogik als Subdisziplin der Erziehungswissenschaft zuzuordnen, ist nicht selbstverständlich, im Gegenteil: Eher werden beide als eigenständige disziplinäre Gegenpole oder Konkurrenten charakterisiert. Im Anschluss an eine Zusammenschau disziplinärer Verortungen und Verhältnisbestimmungen werden in diesem Beitrag Überlegungen zu der Frage angestellt, wie Allgemeine Didaktik und quantitative Unterrichtsforschung als Teilgebiete der Schulpädagogik zu bestimmen sind.

Schlüsselwörter: Allgemeine Didaktik, Unterrichtsforschung, Lehr-Lern-Forschung, Theorie der Schule, Schulpädagogik
\end{abstract}

\section{General Didactics and Empirical Classroom Research as Branches of School Pedagogy}

\section{Summary}

It is no matter of course to allocate general didactics and empirical (quantitative) classroom research as sub-disciplines to educational science, on the contrary: Both are rather regarded as separate disciplinary antipoles or rivals. Subsequent to a synopsis of disciplinary localizations and definitions of relations, this article considers the question, how general didactics and quantitative classroom research can be specified as sub-disciplines of school pedagogy.

Keywords: general didactics, classroom research, school theory, teaching-learning research, school pedagogy

Allgemeine Didaktik und Unterrichtsforschung unter dem gemeinsamen Dach der Schulpädagogik zu fassen, ist alles andere als selbstverständlich oder trivial. Vielmehr finden sich im wissenschaftlichen Diskurs höchst unterschiedliche Positionen 
zur Verortung von Allgemeiner Didaktik und Unterrichtsforschung und zu ihrem Verhältnis. In erster Linie werden beide als eigenständige disziplinäre Zugänge, auch als Gegenpole oder Konkurrenten gekennzeichnet. Dies gilt vor allem dann, wenn empirische Unterrichtsforschung synonym als (schulbezogene) Lehr-LernForschung verstanden und auf empirisch-quantitative Unterrichtsforschung reduziert wird. Warum und wie Allgemeine Didaktik und empirische Unterrichtsforschung als Teilgebiete der Schulpädagogik zu bestimmen sind, wird im Anschluss an eine Zusammenschau ihrer Verortungen und Verhältnisbestimmungen erörtert und abschließend anhand einer Forschungsperspektive illustriert. ${ }^{1}$

\section{Verortungen}

\subsection{Allgemeine Didaktik und Schulpädagogik}

Wie sich die Allgemeine Didaktik zur Schulpädagogik verhält, oder besser: je nach Position verhalten sollte, ist vielfach gefragt worden (vgl. Hellekamps, 2001), aber kaum eindeutig geklärt: Das Verhältnis sei bis in die Gegenwart „nicht überzeugend aufgearbeitet“ (Eickhorst, 2001, S. 727) bzw. nicht klar bestimmt (Solzbacher, 2002).

1) Differenz: Apel (1990) unterscheidet noch Anfang der 1990er-Jahre die Allgemeine Didaktik von der Schulpädagogik und spricht explizit von zwei - eng verbundenen - Disziplinen. Die Didaktik befasse sich, so Apel und Grunder (1995), mit der begrifflichen Analyse des Unterrichts sowie mit der Entwicklung von Planungsvorgaben; die Schulpädagogik sei dagegen eher am Prozess des Unterrichts und an den Handlungsbedingungen, -möglichkeiten und -notwendigkeiten interessiert. Auch in der Gegenwart findet sich im Handbuch Erziehungswissenschaft (Hanke \& Seel, 2015) die explizite Bestimmung von Allgemeiner Didaktik und Schulpädagogik als zwei eigenständigen Disziplinen (vgl. Rothland, 2008).

2) Ausweitung der Allgemeinen Didaktik zur Schulpädagogik: Allgemeine Didaktik wird auch als Vorläuferbezeichnung von Schulpädagogik genannt. Gesprochen wird von einem Wandel Allgemeiner Didaktik bzw. Unterrichtslehre hin zur Schulpädagogik (vgl. Einsiedler, 1995; Ofenbach, 2011), die eine Konzentration auf Unterricht überwinde und Fragestellungen der Didaktik in den größeren Zusammenhang der Schultheorie stelle (Einsiedler, 1995; vgl. auch Bohl, Harant \& Wacker, 2015).

1 Ich konzentriere mich im Folgenden auf die quantitative Unterrichtsforschung, da qualitative Unterrichtsforschung bereits im Sinne einer breiteren, schulpädagogischen Perspektive über das Lehren und Lernen im und die fachbezogene Wirkung von Unterricht hinausgeht. Sie wird auch einvernehmlicher in der Schulpädagogik verortet (vgl. Tillmann, 2005), geht aber keinesfalls in Allgemeiner Didaktik auf (vgl. Helsper \& Klieme, 2013). 
3) Allgemeine Didaktik als Teilbereich der Schulpädagogik: Im gegenwärtigen schulpädagogischen Selbstvergewisserungsdiskurs wird Allgemeine Didaktik in der Regel infolge des unter (2.) angeführten Prozesses als „wesentlicher Bestandteil“ (Bosse, 2010, S. 666) oder Kern der Schulpädagogik bezeichnet (Ofenbach, 2011; Bohl, Harant \& Wacker, 2015). Eine solche Verortung bleibt aber auch gegenwärtig nicht unwidersprochen (vgl. Coriand, 2017).

4) Synonyme Begriffsverwendung: Schließlich ist auch die synonyme Begriffsverwendung von Allgemeiner Didaktik und Schulpädagogik verbreitet. Gesprochen wird von der traditionellen Doppelbezeichnung Schulpädagogik/Didaktik (Tillmann, 2005; vgl. u. a. Gläser-Zikuda, 2008; Leschinsky, 2008; Kowarsch, 2011).

\subsection{Unterrichtsforschung und Schulpädagogik}

Auch empirische Unterrichtsforschung und schulbezogene Lehr-Lern-Forschung werden häufig synonym verwendet (vgl. Gläser-Zikuda, 2008; Gräsel \& Gniewosz, 2015; Willems, 2016). Wo und wie ist Unterrichtsforschung zu verorten?

1) Interdisziplinäre Verortung: Durchaus geläufig ist es, die quantitative Unterrichtsforschung als bedeutsamen Teil der Empirischen Bildungsforschung zu bezeichnen (vgl. u.a. Gräsel \& Gniewosz, 2015; Semper, Mende \& Berkemeyer, 2017). Diese Kennzeichnung steht jedoch nicht im Widerspruch dazu, Unterrichtsforschung auch in der Schulpädagogik anzusiedeln, handelt es sich doch bei der Empirischen Bildungsforschung um ein per defintionem interdisziplinäres Forschungsfeld, zu dem auch Schulpädagogik beiträgt. Gräsel geht hingegen davon aus, dass die Unterrichtsforschung keine Teildisziplin der Erziehungswissenschaft (Gräsel, 2006) und damit der Schulpädagogik sein könne, weil sie interdisziplinär sei. Hier liegt offenbar ein Missverständnis vor, ist doch Disziplinarität die Voraussetzung für Interdisziplinarität.

2) Disziplinäre Verortungen: Wenn es um eine disziplinäre Zuordnung geht, dann werden „Lehr-Lernforschung wie die Unterrichtsforschung [...] aus mehreren Gründen eher der Pädagogischen Psychologie zugeordnet" (Gläser-Zikuda, 2008, S. 192). Dies liege u.a. daran, dass die genutzten theoretischen Ansätze aus der Lern- und Motivationspsychologie stammen und die quantitative forschungsmethodische Ausrichtung dominiere (ebd.; vgl. Gräsel \& Gniewosz, 2015). Schulpädagogik sei dem entgegen weniger für eine empirische Forschungsperspektive offen. Gesprochen wird auch von einem „unerquicklichen Gegeneinander" (Grunder, 2010, S. 34) von Unterrichtsforschung und Schulpädagogik. Gläser-Zikuda fordert gleichwohl alternativ eine Verortung der Unterrichtsforschung als Teildisziplin der Erziehungswissenschaft (Gläser-Zikuda, 2008; so auch Willems, 2016). Schließlich wird Unterrichtsforschung - wie die Allgemeine Didaktik - als zentrales Inhaltsfeld der Schulpädagogik präsentiert (Bohl, Harant \& Wacker, 2015). 
3) Schulpädagogik als Vermittlungswissenschaft: Unterrichtsforschung kann zudem in einem höchst traditionsreichen Verständnis mit der Schulpädagogik verbunden werden, indem diese als Vermittlerin und Umschlagplatz für die Befunde empirischer Forschung verstanden wird. So hieß es etwa in der ersten Auflage der Schul-Pädagogik des Breslauer Seminardirektors Carl Barthel von 1839, dass die Schulpädagogik nicht die Wissenschaft fördern, „sondern die herrlichen Resultate derselben in möglichst weitem Kreise verbreiten" solle (Barthel, 1839, S.7). Auch gegenwärtig wird Schulpädagogik als Sammelpunkt und Übersetzerin von Forschungsbefunden für die Schulpraxis verstanden (vgl. Wellenreuther, 2011; Kowarsch, 2011). Schulpädagogik wird so als eine Subdisziplin gekennzeichnet, die selbst nicht forscht, sondern die Forschungsergebnisse anderer „zum Zweck der Ausbildung und Prüfung angehender Lehrer" vermittelt (Terhart, 2003, S. 192, 200) oder für die Schulpraxis aufbereitet.

Insgesamt kann festgehalten werden, dass - im Vergleich zur Verortung der Allgemeinen Didaktik - bezogen auf die Zugehörigkeit der quantitativen Unterrichtsforschung zur Schulpädagogik weit weniger Konsens herrscht.

\section{Verhältnisbestimmungen}

\subsection{Allgemeine Didaktik vs. empirische Unterrichtsforschung}

Die erste Verhältnisbestimmung von Allgemeiner Didaktik und quantitativer Unterrichtsforschung ist wohl die gängigste: Sie fokussiert auf Gegensätze und Konkurrenz (vgl. Trautmann, 2016). Was aber trennt Allgemeine Didaktik und Unterrichtsforschung? Hier sollen nun nicht die mittlerweile hinlänglich beschriebenen Unterscheidungsmerkmale, Gegensätze, aber auch Gemeinsamkeiten von Allgemeiner Didaktik und quantitativer Unterrichtsforschung bzw. schulbezogener Lehr-LernForschung als „fremde Schwestern“ wiederholt werden (vgl. Terhart, 2002; Bohl, 2004). Stattdessen wird allein (1.) auf die Bedeutung der Unterrichtsinhalte und ihre Bestimmung sowie (2.) auf den Praxisbezug fokussiert, bevor (3.) auf den Ansatz, die Allgemeine Didaktik als hilfreiche Ergänzung der Unterrichtsforschung zu konzipieren, eingegangen wird.

1) In der Allgemeinen Didaktik gehe es vor allem um die Auswahl und Begründung der Unterrichtsinhalte und Bildungsziele, während Unterrichtsforschung eine datenbasierte empirische Beschreibung und Erklärung schulischer Prozesse und ihrer Wirkungen zum Gegenstand habe (Willems, 2016). Lerninhalte und die Lehrenden - als Adressaten - stünden im Vordergrund der Allgemeinen Didaktik, nicht die Lernprozesse und die Lernergebnisse (Gräsel, 2006). 
2) Während Schulpädagogik und in ihr die Allgemeine Didaktik „als Handlungswissenschaft oder Praktische Diszipin“ gilt (Eickhorst, 2001, S. 737), sei als weiteres Unterscheidungsmerkmal eine Orientierung an der Praxis bei Studien der Unterrichtsforschung nicht gegeben. Insofern sei die Idee des Transfers auch nicht in den entsprechenden Untersuchungen mit angelegt (vgl. Gläser-Zikuda, 2008).

3) Vor dem Hintergrund der beanspruchten Praxisorientierung und -relevanz der Allgemeinen Didaktik, aber auch der Schulpädagogik (vgl. Apel \& Sacher, 2009; Fend, 2008), bestehe eine „wesentliche fruchtbare Ergänzung zum psychologischen Ansatz der Unterrichtsforschung [...] darin, dass die Allgemeine Didaktik sich direkt an die unterrichtende Person wendet" (Hasselhorn, 2016, S. 191). Allgemeine Didaktik könne die Unterrichtsforschung insofern unterstützen, als dass sie bei der Implementation forschungsbasierter Innovationen in der Unterrichtspraxis helfe (vgl. Gräsel, 2006). Unterrichtsforschung selbst bleibe so die Domäne der schulbezogenen Lehr-Lern-Forschung.

\subsection{Allgemeine Didaktik als empirische Unterrichtsforschung}

An Stelle eines Neben- oder Gegeneinanders ist der Ansatz einer empirischen Forschungsorientierung der Allgemeinen Didaktik selbst als Reaktion auf die Diagnose ihres Empiriedefizits zu deuten (vgl. Bohl, 2004; Rothland, 2013). Plädiert wird für eine empirische Fundierung Allgemeiner Didaktik, für eine empirische Didaktik als Forschungsprogramm.

Führt die empirische Neuausrichtung aber dazu, Allgemeine Didaktik als empirische Unterrichtsforschung zu entwerfen, droht durch eine solche Angleichung der Identitätsverlust. Das Programm einer allgemeindidaktischen Forschung wäre letztlich keine eigenständige Forschung mehr, sondern sie würde sich selbst durch Kopie der Begriffe, Methoden, Herangehensweisen oder Bewertungsmaßstäbe und Kriterien auflösen. Diese Gefahr konkretisiert sich zum Beispiel in der Übernahme des zentralen Qualitätskriteriums quantitativer Unterrichtsforschung, wenn die Überprüfung der Lernwirksamkeit didaktischer Ansätze als empirische Herausforderung der Allgemeinen Didaktik ausgewiesen wird (vgl. Bohl \& Kleinknecht, 2009; Blömeke \& Müller, 2008).

Im Rahmen allgemeindidaktischer Forschung kann nicht sinnvollerweise von einer Inputorientierung traditioneller Allgemeiner Didaktik auf eine Outputorientierung der schulbezogenen Lehr-Lern-Forschung umgestellt werden, ohne dass damit letztlich Gestalt und Identität der Allgemeinen Didaktik bis zur Unkenntlichkeit verändert werden. 


\subsection{Empirische Unterrichtsforschung als Allgemeine Didaktik}

Schließlich ist auf die umgekehrte Variante einer Verhältnisbestimmung einzugehen: die Darstellung empirischer Unterrichtsforschung als Allgemeine Didaktik. Diese deutet sich eher allgemein an, wenn bspw. Hatties Studie Visible Learning als allgemeindidaktische Theorie bezeichnet wird (vgl. Beywl \& Zierer, 2013, S. XI).

Viel konkreter wird die Vereinnahmung von quantitativer Unterrichtsforschung als Allgemeine Didaktik, wenn das Angebots-Nutzungs-Modell als Modell der Allgemeinen Didaktik neben dem Berliner und Hamburger Modell oder aber Klafkis Perspektivschema der Unterrichtsplanung gestellt wird. „Ein heute sehr prominentes und viel diskutiertes Modell der allgemeinen Didaktik, welches Erkenntnisse der Lernforschung berücksichtigt, ist das Angebots-Nutzungs-Modell“ (Hanke \& Seel, 2015, S. 866; so auch Meyer, 2016).

Als ein zweites, jedoch weniger verwegenes Beispiel kann die fünfte Ausgabe des Jahrbuchs für Allgemeine Didaktik dienen, das sich dem Thema Klassenmanagement/ Klassenführung widmet. Die Herausgeber problematisieren in ihrer Einleitung, dass sich die Allgemeine Didaktik kaum mit dem Thema Klassenmanagement befasst habe (vgl. Haag, Kiel \& Trautmann, 2015, S. 10). Die einzelnen Beiträge des Jahrbuchs für Allgemeine Didaktik weisen selbst jedoch auch keine expliziten Ansätze zu einer spezifisch allgemeindidaktischen Perspektive auf Klassenführung auf. Womöglich sollte hier vor allem ein geradezu klassischer Bereich quantitativer Unterrichtsforschung für die Allgemeine Didaktik beansprucht werden. In den nun folgenden Perspektiven wird eine allgemeindidaktische Perspektive auf Klassenführung erneut aufgegriffen.

\section{Perspektiven}

\subsection{Allgemeine Didaktik als Reflexionsinstanz}

Wie können nun Allgemeine Didaktik und quantitative Unterrichtsforschung jenseits einer konkurrierenden Entgegenstellung oder wechselseitiger Vereinnahmungen zusammen entworfen werden? Und wie ist dies sinnvollerweise unter dem gemeinsamen Dach der Schulpädagogik zu denken? Eine Grundlage für die Verbindung von Allgemeiner Didaktik und Unterrichtsforschung kann im jüngst vorgelegten Entwurf der Allgemeinen Didaktik als Reflexionsinstanz von Rucker (2017) gesehen werden. Rucker sieht die Allgemeine Didaktik nicht nur mit einem Theorie- und Empirieproblem konfrontiert, sondern auch mit einem Reflexionsproblem. Dies resultiere aus dem üblichen Verständnis von Allgemeiner Didaktik als Beschreibungsinstanz von Unterricht. Unter Beschreibung werden hier alle Versuche verstanden, Unterricht unter einer spezifischen, also auch analytischen, forschenden Perspektive 
darzustellen. Dazu zählen u.a. auch Aussagen über die Ziele und Aufgaben von Unterricht (ebd.).

Eine Allgemeine Didaktik als Reflexionsinstanz habe nun nicht den Unterricht als Forschungsgegenstand im Visier, sondern die Voraussetzungen, die der (wissenschaftlichen) „Beschreibung von Unterricht zugrunde liegen“ (ebd., S.621; Herv. i. Orig.). So kann bei der Beschreibung von Unterricht von der Voraussetzung ausgegangen werden, dass es das Ziel sei, Humankapital zu erzeugen. Dann kann aber nicht zugleich von der Voraussetzung ausgegangen werden, schulischer Unterricht habe der Ort zweckfreier Bildung zu sein (ebd.).

Diese und weitere Voraussetzungen der Beschreibung von Unterricht werden in der Allgemeinen Didaktik wenig thematisiert oder hinterfragt, sondern stillschweigend als zutreffend - beansprucht. Allgemeine Didaktik als Reflexionsinstanz problematisiert nun das, was als unproblematisch und selbstverständlich gilt. Während Rucker (2017) diese Funktion vor allem selbstreferenziell auf die Allgemeine Didaktik bezieht, läge hier auch im Rahmen der Schulpädagogik ein weitergehendes Potenzial der Allgemeinen Didaktik für die quantitative Unterrichtsforschung, was am Beispiel der Klassenführung angedeutet werden kann.

Klassenführung als Teil der sogenannten Tiefenstruktur, als Grunddimension, der Unterrichtsqualität hat auch Berührungspunkte mit den Gegenstandsbereichen der Allgemeinen Didaktik. Denn es geht letztlich um die Etablierung, Begründung (etwa gegenüber den Schüler*innen) und Durchsetzung von Normen für die Interaktion und das Miteinander im Klassenzimmer bzw. in der Lehr-Lernsituation. Gerade die Legitimation von Normen (welche Normen vor dem Hintergrund welcher Ziele?), die Bewertung von Verhalten, das als angemessen oder unangemessen bzw. störend zu gelten hat, die Frage, wie Führung und Kontrolle in welchem Maße vor dem Hintergrund der Selbstbestimmungsinteressen der Schüler*innen zu legitimieren sind, all das wären Fragen, die letztlich von einer Allgemeinen Didaktik als Reflexionsinstanz gestellt und als Voraussetzungen der Beschreibung von Unterricht, hier von Klassenführung, bearbeitet werden können.

\subsection{Allgemeine Didaktik und empirische Unterrichtsforschung als Teilgebiete der Schulpädagogik}

Über die Allgemeine Didaktik als Reflexionsinstanz hinaus ist die Kokonstruktion von Allgemeiner Didaktik und quantitativer Unterrichtsforschung unter dem gemeinsamen Dach der Schulpädagogik zu begründen. Hier wird an die Differenzierung von Berliner angeschlossen, der „guten Unterricht“ von „effektivem Unterricht“ unterscheidet (Berliner, 1987, 2005; vgl. Fenstermacher \& Richardson, 2005; Terhart, 2010; Kunter \& Trautwein, 2013). Bezogen auf den Unterricht weist "guter Unterricht“ 
Merkmale auf, die normativen Wertvorstellungen folgen, die als sinnvoll und wünschenswert erachtet werden. „Effektiver Unterricht“ zeichnet sich dadurch aus, dass Lernziele erreicht werden und Unterricht wirkt.

Es erscheint sinnvoll, Berliners Verständnis vom "guten Unterricht“ zu erweitern (er selbst bezieht sich vor allem auf das Lehrerverhalten gegenüber den Schülerinnen). Denn die Unterscheidung von „gutem“ und „effektivem“ Unterricht korrespondiert damit, dass „dem effizient zu instruierenden Individuum der Lehr-Lern-Forschung [...] in der Didaktik ein Subjekt gegenüber [steht], das zur mündigen Partizipation an Gesellschaft erzogen werden soll“ (Wieser, 2013, S. 99). Ein qualitätsvoller Unterricht wäre mit Berliner ein Unterricht, der beiden Normen bzw. Zielen entspräche.

In der getroffenen Unterscheidung käme auch die Allgemeine Didaktik als Reflexionsinstanz in Anbetracht einer quantitativen Unterrichtsforschung zum Tragen, die eher auf die Effektivität sieht als darauf, ob im Rahmen eines effektiven Unterrichts die Interessen und Orientierungen der Heranwachsenden, ihre (auszubildende) Autonomie und Selbstbestimmung berücksichtigt werden. Gerade die Bezugnahme auf die Selbstbestimmungs- und Entfaltungsinteressen der Schüler*innen kann hier als zentraler Fokus eines erweiterten Verständnisses vom „guten Unterricht“ angesehen werden. Zwar wird auch durch die schulisch zu erwerbende Fachkompetenz die Voraussetzung für das selbstbestimmte Handeln in der Gesellschaft geschaffen. Bezogen auf den "guten“ Unterricht geht es aber auch um die Selbstbestimmungsund Entfaltungsinteressen im Unterricht. Erst in der skizzierten Kombination mit der Allgemeinen Didaktik erhält quantitative Unterrichtsforschung ihre schulpädagogische, oder auch allgemeiner, ihre erziehungswissenschaftliche Prägung.

Da sowohl die normativen Wertvorstellungen als auch die Lernziele historisch und gesellschaftlich bedingt sind, bietet die Schulpädagogik zudem den Rahmen der Theorie der Schule und hebt so auch die Institutionenblindheit schulbezogener Lehr-Lern-Forschung (vgl. Gräsel, 2006; Terhart, 2017) auf. Bereits 1977 verwies Benner darauf, dass „Unterrichtsinhalte und Unterrichtsmethoden [...] niemals rein didaktische oder lehrplantheoretische Gegenstände, sondern immer zugleich schultheoretische" seien (Benner, 1977, S.90). Ebendas wäre auch auf die quantitative Unterrichtsforschung zu übertragen: Fragen nach dem Lehren und Lernen im Unterricht sind nie allein über die empirische Erfassung von Prozess- und Produktmerkmalen des Unterrichts zu bestimmen, sondern immer auch allgemeindidaktisch (vor allem mit Blick auf die Begründung der Inhalte des Unterrichts und normativer Wertvorstellungen) und schultheoretisch. Kennzeichen einer schulpädagogischen Unterrichtsforschung wäre damit die Integration einer gesellschaftlichen und individuellen Perspektive (vgl. Bohl, Harant \& Wacker, 2015). 
Allgemeine Didaktik und empirische Unterrichtsforschung in der Schulpädagogik |

Abb. 1: Kokonstruktion von Allgemeiner Didaktik und quantitativer Unterrichtsforschung als Teilgebiete der Schulpädagogik

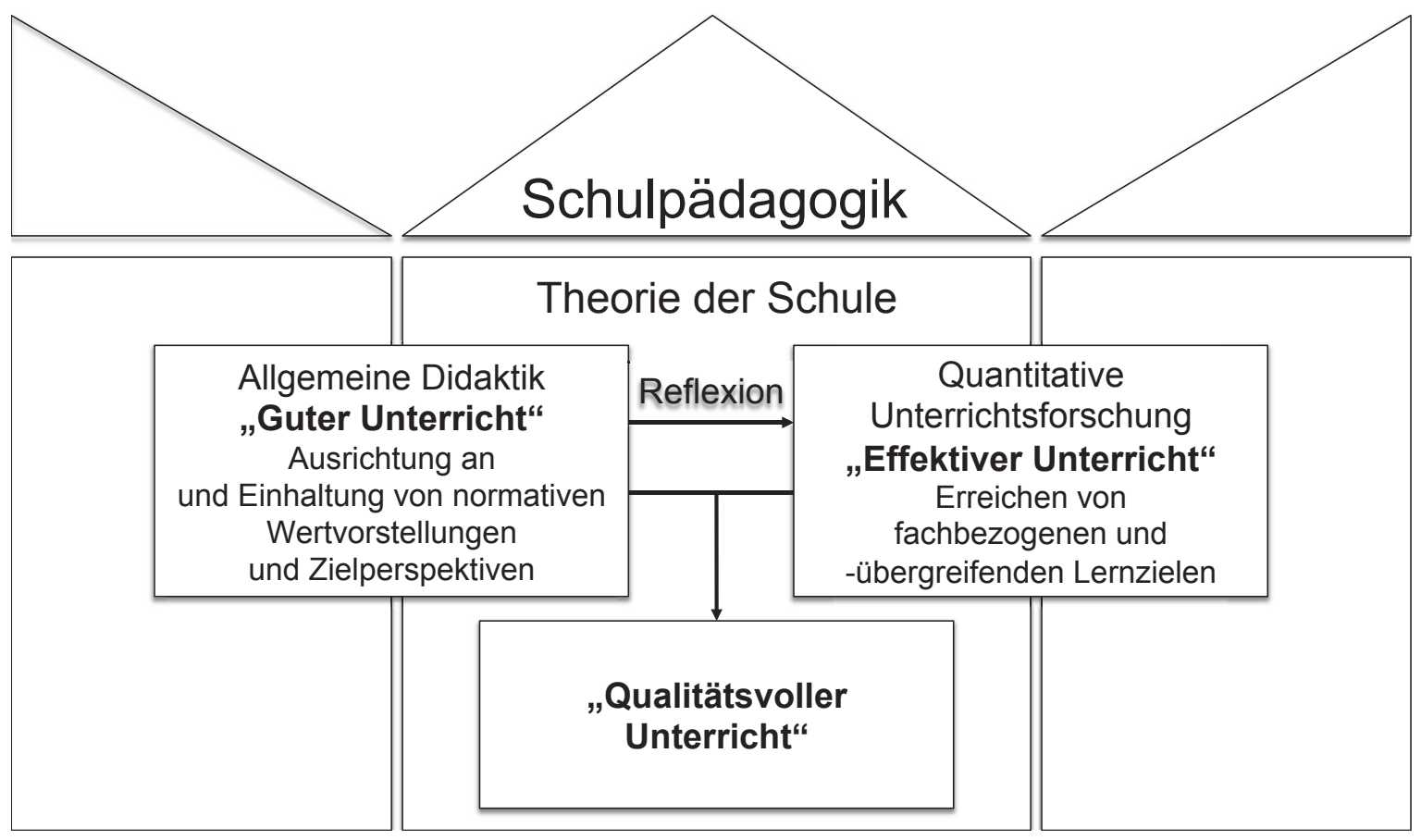

Quelle: eigene Darstellung

\subsection{Forschungsperspektive}

Die Frage nach dem Über- und Außerfachlichen, nach den Selbstbestimmungsund Entfaltungsinteressen der Schüler*innen, nach Autonomie und Partizipation im Unterricht (= ,guter Unterricht") überschreitet die Arbeits- und Kompetenzbereiche der Fachdidaktiken ebenso wie die der quantitativen Unterrichtsforschung. Fachübergreifende Wertorientierungen, Inhalte und Zielstellungen, die gekoppelt sind an gesellschaftliche Wert- und Normvorstellungen sowie an die gesellschaftlichen und individuellen Funktionen von Schule und Unterricht (Enkulturation, Integration), bleiben in einer domänenspezifischen empirischen Unterrichtsforschung zwangsläufig unterbelichtet. Sie werden aber auch gegenwärtig nicht von der Allgemeinen Didaktik behandelt - zumindest nicht über den Rekurs auf die bildungstheoretische Didaktik im engeren Sinne hinaus (vgl. Rothland, 2013).

Dabei können doch gerade die generischen Ziele und Kennzeichen von Unterricht als das schulpädagogische Terrain Allgemeiner Didaktik ausgemacht werden (vgl. Terhart, 2011). Normativ abgeleitete Sollens-Aussagen für den „guten Unterricht“ implizieren auch schulpädagogische Unterrichtsforschung zum Zwecke der Überprüfung intendierter Wirkungen („effektiver Unterricht“) im Sinne eines im Ergebnis qualitätsvollen Unterrichts. 
Welche fachübergreifenden Lerninhalte und -ziele sind es, die in dieser Forschungsperspektive im Kontext der verschiedenen Unterrichtsfächer in den Blick genommen werden sollen? Allgemeinbildende Unterrichtsziele sensu Klafki zielen auf die Selbstbestimmungs-, Mitbestimmungs- und Solidaritätsfähigkeit der Schüler*innen ab (Klafki, 1995). Der Anschluss der hier in Rede stehenden Forschungsperspektive kann jedoch nicht allein an Klafki und die bildungstheoretische Tradition Allgemeiner Didaktik gesucht werden, sondern

1. auch an die individuellen Funktionen der Schule in Fends Neuer Theorie der Schule (Fend, 2009),

2. an die aus den Reihen der quantitativen Unterrichtsforschung von Weinert formulierten fundamentalen Bildungsziele (Weinert, 2000) oder schließlich

3. an die Konzeption und Entwicklung der Bildungsstandards (KMK, 2004/2005).

Als distale Merkmale sind Emanzipation und Autonomie als Zielkategorien einem unmittelbaren empirischen Zugriff kaum zugänglich. Gleichwohl lassen sich konkrete Erhebungsoptionen für proximale Ausprägungen bzw. Facetten fachübergreifender Lernziele und -inhalte benennen: so zur Erfassung der Schülerpartizipation (Kunter, 2005), der Verantwortungsübernahme oder -abwehr sowie der Diskursund Mitbestimmungsmöglichkeiten (vgl. Abs, Diedrich, Sickmann \& Klieme, 2007). Relevant für die empirische Überprüfung fachübergreifender Lernziele und -inhalte können des Weiteren Skalen zur Erfassung der Selbstbestimmung von Schüler*innen (Jerusalem, Drössler, Kleine, Klein-Heßling, Mittag \& Röder, 2009) oder der Autonomie im Sinne von Selbstverantwortlichkeit, Unabhängigkeit und Selbstregulation (vgl. Tönnies, Plöhn \& Krippendorf, 1996) sein. Damit soll deutlich gemacht werden, dass sich die fachübergreifenden Lerninhalte und -ziele nicht per se einem quantitativen Zugriff entziehen.

Im Sinne der hier entworfenen Kokonstruktion von Allgemeiner Didaktik und quantitativer Unterrichtsforschung als Teilgebieten der Schulpädagogik wären bilanzierend folgende Fragen für die skizzierte Forschungsperspektive tragend:

Die leitende Frage wäre (1.), inwieweit Unterricht Selbstbestimmungs- und Entfaltungsinteressen der Schüler*innen achtet, Autonomie und Partizipation fördert („guter Unterricht“) und die Zielvorstellungen auch nachprüfbar („effektiver Unterricht“) erreicht (= „qualitätsvoller Unterricht").

Darüber hinaus wäre (2.) vor allem danach $\mathrm{zu}$ fragen, unter welchen Gestaltungsprämissen des Unterrichtsprozesses ein lernwirksamer Unterricht auch überfachlichen Zielen nachkommen kann. Von besonderem Interesse wäre konkret das Zusammenspiel der Qualitätsmerkmale eines fachbezogen wirkungsvollen Unterrichts wie Klassenführung, Klarheit und Strukturiertheit oder kognitive Aktivierung mit den fachübergreifenden Wertvorstellungen und Zielen des Unterrichts. Naheliegend wäre es, hier an Merkmale wie Schülerorientierung zu denken (Orientierung an 
Vorwissen und Interessen der Schüler*innen, Mitbestimmung, Mitgestaltung). Wie verhalten sich aber Kounins klassische Merkmale der Klassenführung wie Disziplinierung und Allgegenwärtigkeit (Kounin, 1976) zu den Selbstbestimmungsund Entfaltungsinteressen der Schüler ${ }^{\star}$ innen bzw. zum pädagogischen Handeln im Unterricht als Aufforderung zur Selbsttätigkeit?

Drittens (3.) wäre empirisch zu überprüfen, ob und wie es in Abhängigkeit von der jeweiligen Domäne im fachlich geprägten Unterrichtsalltag gelingt, die fachübergreifenden Lernziele $\mathrm{zu}$ verfolgen und $\mathrm{zu}$ erreichen und welche fachlichen Inhalte und Lehr-Lern-Arrangements in höherem Maße dazu geeignet sind und welche nicht. Werden Selbstbestimmungs-, Mitbestimmungs- und Solidaritätsfähigkeit vielleicht im Geschichtsunterricht eher gefördert als im Physikunterricht - oder umgekehrt? In diesem Sinne wäre systematisch fächervergleichende Unterrichtsforschung konstitutiv für eine allgemeindidaktische, schulpädagogische Perspektive.

\section{Literatur und Internetquellen}

Abs, H. J., Diedrich, M., Sickmann, H., \& Klieme, E. (2007). Evaluation im BLK-Modellprogramm "Demokratie lernen und leben“ - Skalen zur Befragung von Schüler/innen, Lehrer/innen und Schulleitungen. Dokumentation der Erhebungsinstrumente 2006 (Materialien zur Bildungsforschung, Bd.20). Frankfurt a. M.: Gesellschaft zur Förderung Pädagogischer Forschung.

Apel, H. J. (1990). Schulpädagogik. Eine Grundlegung. Köln \& Wien: Böhlau.

Apel, H. J., \& Grunder, H.-U. (1995). Die Schulpädagogik - Selbstverständnis, Entstehung, Schwerpunkte schulpädagogischen Denkens. In H. J. Apel \& H.-U. Grunder (Hrsg.), Texte zur Schulpädagogik. Selbstverständnis, Entstehung und Schwerpunkte schulpädagogischen Denkens (S. 7-34). Weinheim \& München: Juventa.

Apel, H. J., \& Sacher, W. (2009). Schulpädagogik als Wissenschaft. In H. J. Apel \& W. Sacher (Hrsg.), Studienbuch Schulpädagogik (4., durchg. Aufl.) (S.7-25). Bad Heilbrunn: Klinkhardt/UTB.

Barthel, C. (1839). Schul-Pädagogik. Ein Handbuch zur Orientierung für angehende Lehrer und zur Beachtung für junge Theologen, als zukünftige Schulrevisoren. Lissa \& Gnesen: Günther.

Benner, D. (1977). Was ist Schulpädagogik? In J. Derbolav (Hrsg.), Grundlagen und Probleme der Bildungspolitik (S. 88-111). München: Piper.

Berliner, D. C. (1987). Simple Views of Effective Teaching and a Simple Theory of Classroom Instruction. In D. C. Berliner \& B. Rosenshine (Hrsg.), Talks to Teachers (S. 93-110). New York: Random House.

Berliner, D. C. (2005). The Near Impossibility of Testing for Teacher Quality. Journal of Teacher Education, 56 (3), 205-213. https://doi.org/10.1177/0022487105275904

Beywl, W., \& Zierer, K. (2013). Lernen sichtbar machen. Zur deutschsprachigen Ausgabe von „Visible Learning“. In J. Hattie, Lernen sichtbar machen. Überarbeitete deutschsprachige Ausgabe von „Visible Learning“ (S. VI-XXVI). Baltmannsweiler: Schneider Verlag Hohengehren.

Blömeke, D., \& Müller, C. (2008). Zum Zusammenhang von Allgemeiner Didaktik und Lehr-Lernforschung im Unterrichtsgeschehen. In M.A. Meyer, M. Prenzel \& S. 
Hellekamps (Hrsg.), Perspektiven der Didaktik. Zeitschrift für Erziehungswissenschaft, 9. Sonderheft (S. 239-258). Wiesbaden: VS.

Bohl, T. (2004). Empirische Unterrichtsforschung und Allgemeine Didaktik. Ein prekäres Spannungsverhältnis und Konsequenzen im Kontext der PISA-Studie. Die Deutsche Schule, 96 (4), 414-425.

Bohl, T., Harant, M., \& Wacker, A. (2015). Schulpädagogik und Schultheorie. Bad Heilbrunn: Klinkhardt/UTB.

Bohl, T., \& Kleinknecht, M. (2009). Weiterentwicklung der Allgemeinen Didaktik - Theoretische und empirische Impulse aus einer Aufgabenkulturanalyse. In K.-H. Arnold, S. Blömeke, R. Messner \& J. Schlömerkemper (Hrsg.), Allgemeine Didaktik und LehrLernforschung. Kontroversen und Entwicklungsperspektiven einer Wissenschaft vom Unterricht (S. 145-157). Bad Heilbrunn: Klinkhardt.

Bosse, D. (2010). Von Schulkritik bis Unterrichtsforschung - Schulpädagogik als Teildisziplin der Bildungswissenschaften. Pädagogische Rundschau, 64 (6), 661-672.

Coriand, R. (2017). Allgemeine Didaktik. Ein erziehungstheoretischer Umriss (2., aktualisierte Aufl.). Stuttgart: Kohlhammer.

Eickhorst, A. (2001). Schulpädagogik - Strukturlinien und Problemlagen. In L. Roth (Hrsg.), Pädagogik. Handbuch für Studium und Praxis (2., überarb. u. erw. Aufl.) (S. 724-742). München: Oldenbourg.

Einsiedler, W. (1995). Schulpädagogik als empirisch begründete, historisch und systematisch orientierte pädagogische Bereichsdisziplin. In H.J. Apel \& H.-U. Grunder (Hrsg.), Texte zur Schulpädagogik. Selbstverständnis, Entstehung und Schwerpunkte schulpädagogischen Denkens (S. 209-220). Weinheim \& München: Juventa.

Fend, H. (2008). Schule gestalten. Systemsteuerung, Schulentwicklung und Unterrichtsqualität. Wiesbaden: VS.

Fend, H. (2009). Neue Theorie der Schule (2., durchges. Aufl.). Wiesbaden: VS. https://doi. org/10.1007/978-3-531-91788-7

Fenstermacher, G., \& Richardson, V. (2005). On Making Determinations of Quality in Teaching. Teachers College Record, 107 (1), 186-213. https://doi.org/10.1111/j.14679620.2005.00462.x

Gläser-Zikuda, M. (2008). Unterrichtsforschung zwischen Schulpädagogik und Lehr-Lernforschung. Plädoyer für einen Brückenschlag. In I. Esslinger-Hinz \& H.-S. Fischer (Hrsg.), Spannungsfelder der Erziehung und Bildung. Ein Studienbuch zu grundlegenden Themenfeldern der Pädagogik (S.191-204). Baltmannsweiler: Schneider Verlag Hohengehren.

Gräsel, C. (2006). Das Verhältnis von Erziehungswissenschaft und Bildungsforschung aus der Perspektive der Unterrichtsforschung. In H. Merkens (Hrsg.), Erziehungswissenschaft und Bildungsforschung (S.97-108). Wiesbaden: VS. https://doi.org/ 10.1007/978-3-531-90089-6_7

Gräsel, C., \& Gniewosz, B. (2015). Überblick Lehr-Lernforschung. In H. Reinders, H. Ditton, C. Gräsel \& B. Gniewosz (Hrsg.), Empirische Bildungsforschung, Bd. 2 (2. Aufl.) (S. 19-24). Wiesbaden: Springer VS.

Grunder, H.-U. (2010). Unterrichtsforschung und ihre schulpädagogische Rahmung. Ein Versuch, das Verhältnis von Schulforschung und Schulpädagogik neu zu bestimmen. Pädagogische Rundschau, 64 (1), 31-44.

Haag, L., Kiel, E., \& Trautmann, M. (2015). Einführung in den Thementeil „Klassenmanagement/Klassenführung - Perspektiven, Befunde, Kontroversen“. In L. Haag, E. Kiel \& M. Trautmann (Hrsg.), Klassenmanagement/Klassenführung - Perspektiven, Befunde, Kontroversen. Jahrbuch für Allgemeine Didaktik, Bd.5 (S.9-14). Baltmannsweiler: Schneider Verlag Hohengehren. 
Allgemeine Didaktik und empirische Unterrichtsforschung in der Schulpädagogik |

Hanke, U., \& Seel, N. M. (2015). Einzeldisziplinen der Erziehungswissenschaft. In N. M. Seel \& U. Hanke, Erziehungswissenschaft. Lehrbuch für Bachelor-, Master- und Lehramtsstudierende (S. 853-904). Wiesbaden: Springer VS.

Hasselhorn, M. (2016). Zur Interdisziplinarität zukünftiger Unterrichtsforschung. In N. McElvany, W. Bos, H. G. Holtappels, M. M. Gebauer \& F. Schwabe (Hrsg.), Bedingungen und Effekte guten Unterrichts (S. 189-193). Münster et al.: Waxmann.

Hellekamps, S. (2001). Schulpädagogik als erziehungswissenschaftliche Disziplin. In M. Brenke (Hrsg.), Schule erleben. Festschrift für Wilhelm Wittenbruch (S.11-22). Frankfurt a. M.: Lang.

Helsper, W., \& Klieme, E. (2013). Quantitative und qualitative Unterrichtsforschung - eine Sondierung. Zeitschrift für Pädagogik, 59 (3), 283-290.

Jerusalem, M., Drössler, S., Kleine, D., Klein-Heßling, J., Mittag, W., \& Röder, B. (2009). Förderung von Selbstwirksamkeit und Selbstbestimmung im Unterricht. Skalen zur Erfassung von Lehrer- und Schülermerkmalen. Berlin: Humboldt-Universität zu Berlin.

Klafki, W. (1995). „Schlüsselprobleme“ als thematische Dimension einer zukunftsbezogenen „Allgemeinbildung“. Zwölf Thesen. In W. Münzinger \& W. Klafki (Hrsg.), Schlüsselprobleme im Unterricht. Die Deutsche Schule, 3. Beiheft (S.9-14). Weinheim: Juventa.

KMK (Ständige Konferenz der Kultusminister der Länder in der Bundesrepublik Deutschland) (2004/2005). Bildungsstandards der Kultusministerkonferenz. Erläuterungen zur Konzeption und Entwicklung. München \& Neuwied: Luchterhand.

Kounin, J. S. (1976). Techniken der Klassenführung. Bern: Huber et al.

Kowarsch, A. (2011). Schulpädagogik - eine Verständigungsbrücke zwischen Schulforschung und Schulpraxis. In S. Hellekamps, W. Plöger \& W. Wittenbruch (Hrsg.), Schule. Handbuch der Erziehungswissenschaft, Bd. 3. Studienausgabe (S.655-664). Paderborn et al.: F. Schöningh.

Kunter, M. (2005). Multiple Ziele im Mathematikunterricht. Münster: Waxmann.

Kunter, M., \& Trautwein, U. (2013). Psychologie des Unterrichts. Paderborn: Schöningh/ UTB.

Leschinsky, A. (2008). Die Ausdifferenzierung und Weiterentwicklung der Schulforschung seit den 1970er Jahren. In W. Helsper \& J. Böhme (Hrsg.), Handbuch der Schulforschung (2., durchg. u. erw. Aufl.) (S.69-88). Wiesbaden: VS. https://doi.org/ 10.1007/978-3-531-91095-6_3

Meyer, H. (2016). Plädoyer für eine Renaissance der Didaktik. In J. Möller, M. Köller \& T. Riecke-Baulecke (Hrsg.), Basiswissen Lehrerbildung: Schule und Unterricht - Lehren und Lernen (S. 206-227). Seelze: Klett/Kallmeyer.

Ofenbach, B. (2011). Schulpädagogik - eine Theorie schulischer Phänomene für die Praxis. In S. Hellekamps, W. Plöger \& W. Wittenbruch (Hrsg.), Schule. Handbuch der Erziehungswissenschaft, Bd.3. Studienausgabe (S.643-654). Paderborn et al.: F. Schöningh.

Rothland, M. (2008). Allgemeine Didaktik - Disziplinäre Bestimmungen zwischen Willkür und Pragmatismus, Theorie und Praxis. In M. A. Meyer, M. Prenzel \& S. Hellekamps (Hrsg.), Perspektiven der Didaktik. Zeitschrift für Erziehungswissenschaft, 9. Sonderheft (S. 173-185). Wiesbaden: VS.

Rothland, M. (2013). Wiederbelebung einer Totgesagten. Anmerkungen zur Reanimation der Allgemeinen Didaktik. Zeitschrift für Erziehungswissenschaft, 16 (3), 629-645. https:// doi.org/10.1007/s11618-013-0369-1

Rucker, T. (2017). Allgemeine Didaktik als Reflexionsinstanz. Versuch einer wissenschaftstheoretischen Grundlegung. Zeitschrift für Pädagogik, 63 (5), 618-635.

Semper, I., Mende, L., \& Berkemeyer, N. (2017). Schul- und Unterrichtsforschung. In T. Burger \& N. Miceli (Hrsg.), Empirische Forschung zum Kontext Schule (S.31-48). Wiesbaden: Springer VS. https://doi.org/10.1007/978-3-658-15437-0_3 
Solzbacher, C. (2002). Systematische Schulpädagogik und ihre möglichen Konsequenzen für die Lehrerbildung. In H. Macha (Hrsg.), Welches Wissen brauchen Lehrer? (S. 66-79). Bad Heilbrunn: Klinkhardt.

Terhart, E. (2002). Fremde Schwestern. Zum Verhältnis von Allgemeiner Didaktik und empirischer Lehr-Lernforschung. Zeitschrift für Pädagogische Psychologie, 16 (2), 77-86. https://doi.org/10.1024//1010-0652.16.2.77

Terhart, E. (2003). Schulpädagogik. Wandlungsprozesse einer Teildisziplin. In M. Fromm \& P. Menck (Hrsg.), Schulpädagogische Denkformen (S. 191-211). Weinheim \& Basel: Beltz.

Terhart, E. (2010). Guter Unterricht: Die Perspektiven der empirischen Unterrichtsforschung und der allgemeinen Didaktik. In C. Fischer \& R. Schilmöller (Hrsg.), Was ist guter Unterricht? Qualitätskriterien auf dem Prüfstand (S. 39-52). Münster: Aschendorf.

Terhart, E. (2011). Allgemeine Didaktik - Fachdidaktik - Lehr-Lern-Forschung. In M. Demantowsky (Hrsg.), Selbstdeutung und Fremdkonzept. Die Didaktiken der kulturwissenschaftlichen Fächer im Gespräch (S. 19-38). Bochum: Projekt-Verlag.

Terhart, E. (2017). Theorie der Schule - eine unendliche Geschichte. In R. Reichenbach \& P. Bühler (Hrsg.), Fragmente zu einer pädagogischen Theorie der Schule. Erziehungswissenschaftliche Perspektiven auf eine Leerstelle (S. 34-53). Weinheim \& Basel: Juventa.

Tillmann, K.-J. (2005). Schulpädagogik und Bildungsforschung. Aktuelle Trends und langfristige Entwicklungen. Die Deutsche Schule, 97 (4), 408-420.

Tönnies, S., Plöhn, S., \& Krippendorf, U. (1996). Skalen zur psychischen Gesundheit (SPG). Testmanual. Heidelberg: Roland Asanger.

Trautmann, M. (2016). Die Allgemeine Didaktik - eine umstrittene Disziplin. In R. Porsch (Hrsg.), Einführung in die Allgemeine Didaktik (S.7-23). Münster et al.: Waxmann.

Weinert, F. E. (2000). Lehren und Lernen für die Zukunft - Ansprüche an das Lernen in der Schule. Pädagogische Nachrichten Rheinland-Pfalz, (2), 1-16.

Wellenreuther, M. (2011). Forschungsbasierte Schulpädagogik. Anleitung zur Nutzung empirischer Forschung für die Schulpraxis. Baltmannsweiler: Schneider Verlag Hohengehren.

Wieser, C. (2013). Konzeptualisierungen von Handeln in Paradigmen der Unterrichtsforschung. Zeitschrift für Pädagogik, 59 (1), 95-111.

Willems, A.S. (2016). Unterrichtsqualität und professionelles Lehrerhandeln. Prozesse und Wirkungen guten Unterrichts aus dem Blickwinkel der empirischen Schul- und Unterrichtsforschung. In R. Porsch (Hrsg.), Einführung in die Allgemeine Didaktik (S. 289337). Münster et al.: Waxmann.

Martin Rothland, Prof. Dr., geb. 1974, Professor für Erziehungswissenschaft mit dem Schwerpunkt Schulpädagogik der Sekundarstufe II an der Universität Siegen.

Anschrift: Universität Siegen, Fakultät II, Adolf-Reichwein-Str. 2a, 57068 Siegen

E-Mail: martin.rothland@uni-siegen.de 